\title{
Pneumonia bacteriana: aspectos epidemiológicos, fisiopatologia e avanços no diagnóstico
}

\author{
Bacterial pneumonia: epidemiological aspects, physiopathology and \\ advances in diagnosis
}

Raíssa Guará Assunção¹, Wellison Amorim Pereira1, Afonso Gomes Abreu³.

\begin{abstract}
Resumo: A pneumonia é uma doença infecciosa que atinge os alvéolos pulmonares, ocasionada, principalmente, por bactérias, vírus, fungos e parasitas. Dentre as principais bactérias, Haemophilus influenzae, Klebsiella pneumonia, Streptococcus pneumoniae, Staphylococcus aureus e as do grupo das Gram-negativas não fermentadoras de glicose, a exemplo de Acinetobacter spp. e Pseudomonas aeruginosa, são as mais isoladas. Estima-se que 450 milhões de casos de pneumonia ocorram por ano em todo o mundo e o Brasil é protagonista na lista dos países com maior número de casos. Pacientes que fazem uso de ventilação mecânica têm até 21 vezes mais chances de desenvolver a doença e $20 \%$ daqueles que utilizam a técnica por mais de 48 horas desenvolverão a infecção. Desta forma, esta revisão teve por objetivo fazer uma avaliação crítica sistematizada da literatura sobre a doença, aspectos epidemiológicos, fisiopatologia e avanços no diagnóstico. Este estudo observou que houve uma evolução nos métodos de diagnóstico, resultando na diminuição expressiva do número de mortes. No entanto, para que a queda no número de acometidos se mantenha, a correta prescrição e utilização da antibioticoterapia são imprescindíveis.
\end{abstract}

Palavras chaves: pneumonia; causas; diagnóstico, bactéria multirresistente.

\begin{abstract}
Pneumonia is an infectious disease that reaches the lung alveoli, caused mainly by bacteria, viruses, fungi and parasites. Among the main bacteria, Haemophilus influenzae, Klebsiella pneumonia, Streptococcus pneumoniae, Staphylococcus aureus and those of the nonfermenting group of Gram-negative glucose, for example Acinetobacter spp. and Pseudomonas aeruginosa, are the most isolated. It is estimated that 450 million cases of pneumonia occur every year around the world, and Brazil is a major player in the list of countries with the greatest number of cases. Patients who use mechanical ventilation are up to 21 times more likely to develop the disease and $20 \%$ of those who use the technique for more than 48 hours will develop the infection. Thus, this review aimed to make a systematic critique of the literature on the disease, epidemiological aspects, pathophysiology and advances in diagnosis. This study observed that there was an evolution in the diagnostic methods, resulting in a significant decrease in the number of deaths. However, for the decrease in the number of affected patients to be maintained, the correct prescription and use of antibiotic therapy are essential.
\end{abstract}

Key words: pneumonia; causes, diagnosis; multiresistant bacteria.

\footnotetext{
1 Biomédico (a). Mestrando (a) em Biologia Parasitária (UNICEUMA). Laboratório de Microbiologia Ambiental (UNICEUMA).

${ }^{2}$ Farmacêutico. Docente do Mestrado em Biologia Parasitária (UNICEUMA).
} 


\section{Introdução}

As doenças respiratórias afetam crianças, adultos e idosos e são consideradas importantes causas mundiais de morbi/mortalidade. Segundo os dados da Organização Mundial de Saúde, as doenças respiratórias representam aproximadamente $14 \%$ do total de mortes no mundo. Em 2012, de acordo com o World Health Statistics, as principais causas de anos potenciais de vida perdidos no mundo foram devido às doenças cardíacas isquêmicas e as infecções do trato respiratório inferior, a exemplo da pneumonia ${ }^{1}$.

A pneumonia é um processo inflamatório agudo ou crônico do parênquima pulmonar produzido por bactérias, vírus, fungos, parasitas ou outros processos que levem a inflamação ou infecção do aparelho respiratório. Os primeiros relatos da doença remontam ao ano de 1250 A.C em múmias encontradas no Egito e desde então vem crescendo e sendo disseminada pelo mundo².

É sabido que dentre os microrganismos associados à pneumonia, as bactérias são os principais; porém, pouco se sabe sobre os padrões patológicos dos microrganismos envolvidos. A maior parte dos estudos está direcionada a evolução clínica e tratamento ${ }^{3}$.

\section{Método de pesquisa}

Trata-se de uma revisão de literatura sistemática realizada a partir de amplo levantamento, avaliação e seleção de artigos relevantes publicados nos últimos anos. Os dados pertinentes a esta pesquisa foram extraídos dos principais periódicos científicos nacionais e internacionais, como
Scielo, PubMed (US National Library of Medicine /National Institutes of Health) e LILACS. Foram utilizadas as seguintes palavras chave para a triagem dos artigos: "pneumonia", "diagnosis of pneumonia", "causes of pneumonia", "epidemiology of pneumonia", "multidrug resistant bacteria".

Os principais critérios de inclusão foram: relevância dos dados, fonte e ano de publicação. Assim, um total de 60 artigos foram encontrados e destes, após avaliação crítica do conteúdo dos trabalhos, um total de 36 foram selecionados.

\section{Resultados}

Este estudo observou que houve uma evolução nos métodos de diagnóstico, resultando na diminuição expressiva do número de mortes causadas pela doença nos últimos anos. Porém, a América Latina ainda concentra os países com maior número de doentes, estando o Brasil no primeiro lugar do ranking. Estudos recentes mostram que um dos principais grupos de risco para o desenvolvimento da pneumonia são o dos pacientes internados em UTI, principalmente idosos e crianças. Assim, para que a queda no número de casos de pneumonia se mantenha, a correta prescrição e utilização da antibioticoterapia é imprescindível, visando a redução nos casos de multirresistência.

\section{Discussão}

\section{Epidemiologia}

Nos últimos anos os avanços nas técnicas de diagnóstico 
proporcionaram uma queda expressiva no número de casos de pneumonia, no entanto, a doença ainda figura entre as principais causas de morbimortalidade mundial ${ }^{3}$.

Dados revelam que cerca de 450 milhões de pessoas são infectadas pela doença todos os anos no mundo e até 1,5 milhão de crianças menores de cinco anos são acometidas pela pneumonia apenas na América Latina. Estima-se que a doença mata cerca de 4 milhões de pessoas anualmente ${ }^{4,5}$.

O Brasil é protagonista na lista dos países com maior número de casos (4). Segundo informações geradas a partir do Sistema de Informação sobre Mortalidade do Departamento de Tecnologia da Informação do Sistema Único de Saúde (SIM/DATASUS), entre os anos de 1996 e 2002, a pneumonia foi responsável por $37 \%$ dos óbitos relacionados ao sistema respiratório ${ }^{6}$.

Um levantamento feito pelo National Nosocomial Infection Surveillance (NNIS) do Center for Disease Control and Prevention (CDC) revelou que a incidência da doença em pacientes em uso de ventilação mecânica chega a ser até 21 vezes maior do que em outros pacientes que não fazem uso de ventilação mecânica7. Um estudo realizado por Bezerra et al. ${ }^{7}$ mostrou que até $20 \%$ daqueles que utilizam a técnica por mais de 48 horas desenvolverão a infecção.

\section{Fisiopatologia}

A pneumonia é um grave problema de saúde pública associado a alta morbidade e mortalidade, resultando em um processo infeccioso das vias aéreas inferiores através da aspiração ou inalação de microrganismos patogênicos ${ }^{8}$.

Ao chegar ao pulmão, os microrganismos envolvidos colonizam e invadem a região. Em indivíduos imunocomprometidos, até mesmo bactérias de média e baixa virulência podem estar relacionadas. Assim, levam a um quadro de infecção do parênquima pulmonar, região importante para as trocas gasosas de competência do sistema respiratório. Logo, os bronquíolos e alvéolos são preenchidos por exsudato inflamatório, dificultado a hematose e levando ao quadro clássico de insuficiência respiratória 9 .

Elementos estruturantes das bactérias, como flagelo, fímbrias e polissacarídeos, tornam o processo de adesão mais ágil e preciso. No epitélio do sistema respiratório, levam a adesão e, em alguns casos, a consecutiva infecção ${ }^{10}$. Em geral, esses agentes patogênicos originam-se principalmente da microbiota endógena do paciente e de indivíduos próximos a ele. Quando oriundos de outras fontes, advém especialmente das mãos dos profissionais de saúde e materiais/equipamentos hospitalares não estéreis ${ }^{11}$.

\section{Microrganismos envolvidos}

A colonização bacteriana no trato respiratório é desenvolvida notoriamente por bactérias como Haemophilus influenzae, Streptococcus pneumoniae, Staphylococcus aureus, Acinetobacter spp. e Pseudomonas aeruginosa, todas comumente isoladas em Unidades de Terapia Intensiva (UTI). Além destas, outras 
bactérias também são relacionadas à infecção em ambiente hospitalar via método de respiração mecânica. São normalmente microrganismos com baixa exigência nutricional, com larga expressão de fatores de virulência, aeróbios e não esporulados. Por tais características, sobrevivem até mesmo às condições mais extremas, a exemplo de Klebsiella pneumoniae.

Dentre os principais encontrados em UTI estão os bacilos Gram-negativos não fermentadores, como Acinetobacter spp e $P$. aeruginosa. Esses microrganismos são aeróbios e não esporulados, sendo capazes de sobreviver por longo período devido a sua escassa exigência nutricional e à presença de diversos fatores de virulência. A fonte de contágio dessas bactérias, geralmente, é a ambiental, bem como dos equipamentos de respiração, umidificadores, colchões e travesseiros ${ }^{12}$.

Assim também, quanto às taxas de mortalidade de algumas bactérias em pacientes em uso de ventilação mecânica $(\mathrm{VM})$, alguns autores diferem. A maioria destes afirma que, quando a pneumonia está associada à Pseudomonas spp. e Acinetobacter spp., a mortalidade destes pacientes aumenta. Com relação a um estudo feito em 2017, a VM é protagonista neste quesito, pois até $90 \%$ dos que a utilizam, desenvolvem a patologia ${ }^{13}$.

Tal afirmação baseia-se nas características destas bactérias. 0 gênero Acinetobacter, por exemplo, compreende cerca de 31 espécies diferentes, porém, a espécie que mais coloniza a pele humana é o Acinetobacter baumannii. Essa bactéria está envolvida em processos infecciosos, não só em pacientes que possuem queimaduras como também nos que estão em UTI. Ademais, a bactéria possui características que a torna difícil de ser tratada. Destaque para seu caráter multirresistente a drogas antimicrobianas e fácil transmissão, sendo comumente isolada em UTI. Deste modo, o controle de sua proliferação e transmissão em ambiente hospitalar consiste nas boas práticas de higiene de equipamentos hospitalares ${ }^{14}$.

Igualmente, um estudo recente levantou a discussão acerca da associação entre $A$. baumannii e a formação de biofilme. Segundos dados publicados, a formação de biofilme dificulta a eliminação da bactéria de equipamentos usados em estabelecimentos de saúde, a exemplo dos cateteres, materiais plásticos e tubos de ventilação mecânica, aumentando a possibilidade de infecção ${ }^{15}$.

De igual modo, $P$. aeruginosa tem grande relevância para 0 desenvolvimento da pneumonia. Trata-se de uma bactéria do grupo das Gram-negativas, comumente encontrada nas mucosas e na pele, disseminando-se essencialmente via contato. Para pessoas imunocomprometidas, representa grande risco por ter caráter oportunista e fatores de virulência, invasão, liberação de substâncias como endotoxinas e exotoxinas, que dificultam sua eliminação. Além disso, possui a capacidade de superprodução de bombas de efluxo, redução de sua permeabilidade e alta produção de enzimas que degradam os antibióticos; logo, também são microrganismos que facilmente adquirem resistência ${ }^{16,17}$. Ainda no grupo dos microrganismos causadores de pneumonia está a $K$. pneumoniae. Além de atrelada à doença, também é citada como causadora de infecções renais e de pele adquiridas 
em ambiente hospitalar. Constituinte da classe dos Gram-negativos, pertence à família das Enterobacteriaceae, tem forma de bastão e é anaeróbio. De igual modo, vem sido descrita como resistente aos antibióticos carbapenêmicos, penicilinas e cefalosporinas, em função de mecanismos enzimáticos, como a produção de beta-lactamases e carbapenemases ${ }^{18,19}$.

Além destas, outra bactéria de interesse clínico para a pneumonia é - S. aureus. Normalmente são associados a doenças de pele, mas, em casos graves, podem levar a pneumonia e sepse. Quando alcança o sistema respiratório, o microrganismo pode levar a um quadro grave de pneumonia, pois geralmente seu tempo de infecção tende a ser longo, expressando proteínas citotóxicas capazes de agredir tecidos e, por consequência, agravar a doença. Entre os fatores que propiciam a sua infecção, estão: hospitalização por longos períodos, doença(s) de base, imunodeficiência, contato com materiais hospitalares contaminados e exposição a antibióticos por longo período ${ }^{20}$.

\section{Classificação}

A pneumonia pode ser classificada em: Pneumonia Adquirida na Comunidade (PAC), Pneumonia Adquirida em Hospital (PAH) e a Pneumonia Adquirida em Ambiente Hospitalar por Ventilação Mecânica (PAV). Assim, a PAC é uma infecção aguda do parênquima pulmonar, origem comunitária ou que se manifesta em até 48 horas pós-internação ${ }^{21}$.

A PAH é o tipo mais grave e caracteriza-se por só ser diagnosticada

pós-internação, normalmente após 48 de internação. Estima-se que até $1,1 \%$ de todos os pacientes internados a desenvolva e, apesar da baixa incidência, possui mortalidade de até $50 \%$, principalmente quando relacionada a microrganismos multirresistentes, estando associada também a elevação de gastos com internação ${ }^{22}$.

PAV, por sua vez, é considerada a segunda infecção nosocomial mais comum e a principal causa de morte em pacientes em estado críticos pelo caráter invasivo dos procedimentos de ventilação mecânica. Sua relevância clínica torna-a um constante desafio para as equipes de saúde ${ }^{23,17}$. Difere da PAH por seu desenvolvimento ocorrer após 48 horas após a intubação orotraqueal; é subclassificada em PAV precoce, quando se manifesta até 4 dias após o procedimento, e PAV tardia, quando manifesta-se 5 dias após 0 procedimento ${ }^{24}$. O principal meio de transmissão de PAV é sabidamente por meio de profissionais da saúde, especialmente 0 contato com diferentes doentes sem a higienização das mãos, além do uso de materiais e equipamentos respiratórios não estéreis ${ }^{25}$.

\section{Grupos e Condições de Risco}

O grupo de risco para 0 desenvolvimento da pneumonia é formado, principalmente, por crianças, idosos (especialmente os com idade superior a 65 anos e hospitalizados), gestantes, imunocomprometidos, profissionais de saúde e pacientes internados em UTI, de uma forma geral ${ }^{26}$.

O longo período de tratamento dos doentes propicia 
desenvolvimento de infecções nosocomiais. Independente dos grupos de risco, alguns fatores aumentam as chances da infecção, tais como: idade elevada, desnutrição, doenças de base, depressão do nível de consciência, doenças pulmonares e cardiológicas, ventilação mecânica, manipulação do paciente pela equipe hospitalar, uso de sondas ou de cânula nasogástrica, intubação ou reintubação orotraqueal, traqueostomia, macro ou microaspiração de secreção traqueobrônquica, uso prévio de antimicrobianos, trauma grave, broncoscopia e bronco-aspiração de microrganismos da orofaringe, administração de antiácidos ou de bloqueadores de receptores $\mathrm{H} 2$, permanência em posição supina e transporte dentro do hospital ${ }^{24}$.

Dentre estes, os mais nocivos são a Intubação Endotraqueal e a Ventilação Mecânica, procedimentos rotineiros em UTI ${ }^{27}$. O uso de suporte ventilatório revolucionou 0 tratamento da insuficiência respiratória, entretanto, pode gerar efeitos negativos em pacientes com pneumonia, devido à redução das defesas locais frente à presença do tubo endotraqueal ${ }^{28}$.

\section{Profilaxia}

A relevância da doença, singularmente, quando adquirida em ambiente hospitalar por indivíduos em tratamento de outras comorbidades, expressa a necessidade da realização de ações de impacto que levem a redução nos casos de pneumonia. Tais ações devem surgir a partir da análise da fisiopatologia e dados epidemiológicos respeitando as particularidades locais. Hábitos simples como a constante e correta higienização das mãos, manutenção da posição de decúbito em pacientes internados, elevação da cabeceira 30-45, interrupção da sedação, higiene oral do paciente, pressão do "cuff", extrema perícia com o manuseio e assepsia de equipamentos, além do diagnóstico rápido e tratamento eficaz, podem reduzir drasticamente os números de casos anuais de infecção e óbitos por pneumonia $29,27$.

Com relação à $\mathrm{PAV}$, a criação de protocolos de práticas e condutas hospitalares, sobretudo em UTI, se aplicado corretamente, de forma multiprofissional e credenciado por órgão especializado, promoverá redução drástica na incidência da infecção ${ }^{30}$.

\section{Diagnóstico \\ O diagnóstico da infecção se} dá por meio da análise de achados diversos obtidos por meio de evidencias clínicas, laboratoriais e de exames de imagem, como os radiológicos. A radiografia revela presença de infiltrado persistente de caráter recente ou progressivo, cavitação pulmonar ou consolidação. Os exames laboratoriais e a clínica, por sua vez, revelam aspectos como: leucopenia (inferior a $4000 \mathrm{cé} / / \mathrm{mm}^{3}$ ) ou leucocitose (maior que 12000 cel $/ \mathrm{mm}^{3}$ ), febre, secreção purulenta com sua mudança ou aumento gradual ${ }^{31}$.

Além destes, os testes de identificação microbiana também podem ser empregados, visando aprimorar o diagnóstico, já que se sabe que outros achados, de forma isolada, carecem de especificidade ${ }^{31}$. Além do isolamento do patógeno envolvido na infecção, o método também gera informações 
relevantes acerca da sensibilidade dos patógenos aos antimicrobianos. Por fim, têm-se o diagnóstico mais completo e, por consequência, tratamento mais efetivo ${ }^{32}$.

Em 2013, o Center For Disease

Control and Prevetion (CDC) publicou algumas normas para vigilância de eventos associados à ventilação mecânica em adultos. O documento objetiva sanar as dificuldades operacionais envolvidas no diagnóstico e apuração de dados epidemiológicos, em especial, voltado para $\mathrm{PAV}^{33}$.

A demora na liberação de diagnóstico para pneumonia devese, em parte, aos métodos convencionais de diagnóstico e demora no encaminhamento a partir da suspeita clínica. Logo, faz-se necessário o aprimoramento cada vez maior das técnicas empregadas no diagnóstico da infecção, que precisa ser rápida e específica. Acrescenta-se a isto a importância vital exercida pela equipe de saúde, responsável por prestar esclarecimentos ao paciente e orientá-lo quanto às etapas do tratamento. Assim, quanto mais cedo o diagnóstico, mais eficaz será o tratamento, evitando-se o uso errôneo de medicamentos e, por consequência, o desenvolvimento de bactérias resistentes e 0 decréscimo do número de casos de morte por pneumonia ${ }^{21,34}$.

\section{Tratamento}

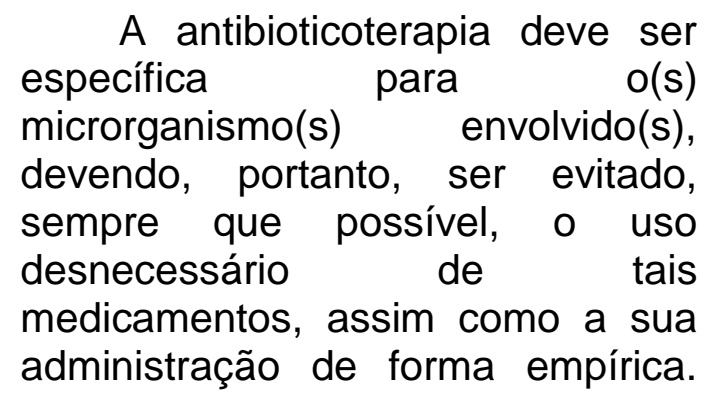

Para pacientes não internados e previamente sadios, é indicado o uso de um macrolídeo. Para aqueles com comorbidades prévias, associação de macrolídeo e um beta-lactâmico ou apenas fluoroquinolona. Pacientes internados, por sua vez, macrolídeo associado a beta-lactâmicos ou fluoroquinolona ${ }^{35}$.

O controle no uso de antimicrobianos durante 0 tratamento é fundamental para evitar surtos. Os principais cuidados pairam sobre o uso exagerado de fluoroquinolonas, carbapenêmicos e cefalosporinas, que devem ser observados. Assim, o entendimento dos mecanismos de resistência responsáveis por esta situação e de práticas adequadas de controle de infecção são fundamentais para manejar melhor essas infecções ${ }^{15}$.

É sabido que todos os antibióticos de algum modo selecionam e, então, favorecem a resistência, levando a mudança da microbiota, de revestida por microrganismos sensíveis para revestida por resistentes. Assim, a emergência do tema levou a Organização Mundial da Saúde (OMS) a reconhecer a Resistência Antimicrobiana como um sério problema enfrentado pela saúde pública global ${ }^{36}$. 


\section{Conclusão}

A pneumonia é uma das doenças infecciosas que mais matam no mundo e o Brasil está entre os países com maior incidência da doença, sendo, ainda mais grave, entre pacientes que fazem uso de ventilação mecânica. Desta forma, faz-se necessário a aplicação de medidas de controle mais eficazes para evitar a disseminação desses microrganismos.

\section{REFERÊNCIAS}

1. Ferraz RO, Oliveira-friestino JK, Francisco PMSB. Tendência de mortalidade por pneumonia nas regiões brasileiras no período entre 1996 e 2012. J. bras. pneumol., São Paulo, v. 43,n. 4,p. 274-279, Aug. 2017.

2. Matoso LML, Castro CHA. Indissociabilidade clínica e epidemiológica da pneumonia. Catussaba, V. 2, n. 2, p.11-23, 2013.

3. Rocha NetoOG, LeiteRF, BaldiBG. Atualização em pneumonia comunitária viral. Rev. Assoc. Med. Bras., São Paulo,v. 59,n. 1,p. 78-84, fev. 2013.

4. Nunes BP, Soares MU, Wachs LS, Volz PM, Saes MO, Duro SMS, et al. Hospitalização em idosos: associação com multimorbidade, atenção básica e plano de saúde. Rev Saude Publica. 2017;51:43.

5. Ruuskanen O, Lahti E, Jennings LC, Murdoch DR. Viral pneumonia. Lancet, 2011.

6. Sociedade de Pediatria do Rio Grande do Sul. Informe Técnico. Campanha Nacional de Vacinação Contra a Influenza, Brasília, 2014.

7. Bezerra EL, Lima AIF, Nóbrega ARR, et.al. Prevalência de pneumonia em pacientes de uma unidade de terapia intensiva de um hospital-escola de Fortaleza - CE. Ver Bras Promoç Saúde. V. 25(2), p. 20-24, 2012.

8. Cilloniz C, Martin-Loeches I, GarciaVidal C, San Jose A, Torres A. Microbial Etiology of Pneumonia: Epidemiology, Diagnosis and Resistance Patterns. Esposito S, ed. International Journal of Molecular Sciences, 2016.

9. Machado D, de Moraes LTAR, de Oliveira ME, Vianna IA. Pneumonia: Tratamento e Evolução. Cadernos UniFOA, 2017.

10. Aykac K, Yasemin O, SevgenTB. Future Directions and Molecular Basis of Ventilator Associated Pneumonia. Canadian Respiratory Journal, 2017. 
11. Albernaz MP, Gonçalves EO, de Lima MS, de Lima Melo J, Pontes MS R, Sousa AOB. Práticas assistenciais de enfermagem e prevenção da pneumonia associada à ventilação mecânica em UTI. Revista de enfermagem, 2015.

12. Carneiro M, Saridakis HO. Pneumonia associada à ventilação mecânica por Acinetobacter baumannii resistente a carbapenem. Rev Panam Infectologia, 2008.

13. Barrada LM, Teixeira MM, da Silva LO, Retamero DF, Maia ABF, Silva KMR, de Barcellos LRNF. Pneumonia associada à ventilação mecânica: avaliação do conhecimento e orientações à profissionais intensivistas. UNILUS Ensino e Pesquisa,V. 14(36), p. 41-55, 2017.

14. Frazão MR. Pesquisa fenotípica de resistência em amostras de Acinetobacter baumannii isoladas em um Hospital de Emergência, 2010.

15. Martins AF, Barth AL. Acinetobacter multirresistente - um desafio para a saúde pública. Scientia Medica (Porto Alegre); v.23,n. 1, p. 56-62, 2013.

16. Cordeiro ALO, et al. Contaminação de equipamentos em unidade de terapia intensiva. Acta paul. enferm.,São Paulo, v. 28,n. 2,p. 160-165, Apr. 2015.

17. Rodrigues, PMA et al. Pneumonia associada à ventilação mecânica: epidemiologia e impacto na evolução clínica de pacientes em uma unidade de terapia intensiva. J. bras.pneumol., São Paulo, v. 35, n. 11, p. 1084-1091, Nov. 2009.

18. Meyer G, PicoliSU. Fenótipos de betalactamases em Klebsiella pneumoniae de hospital de emergência de Porto Alegre.J. Bras. Patol. Med. Lab., Rio de Janeiro,v. 47,n. 1,p. 2431,Feb.2011.

19. Soares, Valéria Martins. Emergência de Klebsiella pneumoniae produtora de carbapenemase (KPC) em um hospital terciário. J. Bras. Patol. Med. Lab., Rio de Janeiro, v. 48, n. 4, p. 251253, Aug. 2012.
20. Perez MG, et al. Factores de riesgo de focos secundarios de infección en ninos con bacteriemia por Staphylococcus aureus adquirida en la comunidad. Enferm Infecc Microbiol Clin, 2017.

21. SBP. Sociedade Brasileira de Pneumologia. Jornal Brasileiro de Pneumologia. V. 38, n. 1, p-s1-s46, abril 2012.

22. Rufino R, Pugliese JG, Kirk K, BartholoTgP, Mafort TT. Pneumonia adquirida no hospital - visão crítica. Revista Hospital Universitário Pedro Ernesto, 2010.

23. Timsit JF, Esaied W, Neuville M, Bouadma L, Mourvllier B. Atualização sobre a pneumonia associada à ventilação. Research, 2017.

24. Amaral SM, Cortes $A Q$, Pires FR. Pneumonia nosocomial: importância do microambiente oral. J. bras. pneumol.,São Paulo, v. 35,n. 11,p. 1116-1124, Nov.2009.

25. Ibn Saied W, et al. Impacto Respectivo da Implementação de Estratégias de Prevenção, Colonização com Bactérias Multi-Resistentes e Uso de Antimicrobianos sobre o Risco de VAP de Início e Tarde: uma Análise da Rede Outcomerea. Ed.Yu Ru Kou.PLoS, 2017.

26. Brasil. Ministério da Saúde. Secretaria de Vigilância em Saúde. Departamento de Vigilância Epidemiológica. Doenças infecciosas e parasitárias: guia de bolso / Ministério da Saúde. Secretaria de Vigilância em Saúde, Departamento de Vigilância Epidemiológica, 8. ed. Ver, Brasília : Ministério da Saúde, 2010.

27. Silva RM. Pneumonia associada à ventilação mecânica: fatores de risco. Rev Bras Clin Med. V. 9(1), p.5-10, 2011.

28. Albernaz MP, Gonçalves EO, de Lima MS, de Lima Melo J, Pontes MS R, Sousa AOB. Práticas assistenciais de enfermagem e prevenção da pneumonia associada à ventilação mecânica em UTI. Revista de enfermagem, 2015. 
29. Almeida KMV, et al. Adesão às medidas de prevenção para pneumonia associada à ventilação mecânica.Revista de Enfermagem da UFSM, [S.I.], v. 5, n. 2, p. 247 - 256, jul. 2015.

30. Gomes AM, Da Silvar CL. Prevenção da pneumonia associada à ventilação mecânica: $O$ que sabem os enfermeiros a esse respeito?. Revista de Pesquisa: Cuidado é fundamental Online, 2010.

31. Dalmora $\mathrm{CH}$, et al. Definindo pneumonia associada a ventilação mecanica: conceito em (des) construção. Revista Brasileira de terapia intensiva, São Paulo, v.25,n.2,p.81-86, jun 2013.

32. Bonten MJM. Ventilator associed pneumonia: preventing the inevitable. Clinical infections diseases. V. 52(1), p.115-121, 2011.

33. Waltrick $R$, et al . Comparação entre um método de diagnóstico clínico e a técnica de vigilância do Center for Disease Control and Prevention para identificação de pneumonia associada à ventilação mecânica. Rev. bras. ter. intensiva, São Paulo ,v. 27,n. 3,p. 260265, Sept. 2015.

34. De Amorim CR, Lundgren FLC, Pereira-Silva JL, Frare RL. Diretrizes brasileiras para pneumonia adquirida na comunidade em adultos imunocompetentes. Jornal Brasileiro de Pneumologia, v. 35(6), p. 574-601, abr 2009.

35. Sociedade Brasileira de Pneumologia e Tisiologia. Diretrizes brasileiras para tratamento das pneumonias adquiridas no hospital e das associadas à ventilação mecânica e Diretrizes brasileiras em pneumonia adquirida na comunidade em pediatria. J Bras Pneumol, 2007.

36. Soldatelli RPP, Lorenzini E. Estratégias para prevenção da resistência bacteriana: contribuições para a segurança do paciente. Revista Cuidarte, [S.I.], v. 5, n. 2, p. 757-64, nov. 2014. 